\title{
Suicide from the Clifton Suspension Bridge in England
}

\author{
Mike Nowers, David Gunnell
}

\begin{abstract}
Objectives - To examine the epidemiology of suicide by jumping from the Clifton Suspension Bridge and its impact on local patterns of suicide.

Design - Case-control study of falls from the bridge (1974-93) matched by age and sex with those using other methods of suicide. Routine OPCS mortality statistics for Bristol and District Health Authority. Setting - The County of Avon and the Bristol and District Health Authority. Subjects - 1. Individuals given coroners' verdicts of suicide, "open", or misadventure after falls from the suspension bridge and 127 matched control suicides using other methods. 2. All deaths from suicide within the Bristol and District Health Authority 1982-91.
\end{abstract}

Main outcome measures - Past psychiatric history, demographic characteristics of suicides, and proximity of place of residence to the bridge.

Results - There were 127 falls from the Clifton Suspension Bridge between 1974 and 1993. The mean age was 35.4 years for males $(n=93)$ and $35 \cdot 5$ for females $(n=34)$. Those who committed suicide by jumping were no more likely to have psychiatric histories than controls $(95 \%$ CI of difference $-1 \cdot 17 \%, 23 \cdot 2 \%$ ) and were no more likely to have been psychiatric inpatients in the past $(95 \%$ CI of difference $-10 \cdot 2 \%$, $13 \cdot 3 \%)$. Mean distance of residence from the bridge differed little between jumping suicides and controls (difference $1.7 \mathrm{~km}$ $95 \%$ CI $0.5,3.9 \mathrm{~km})$. Altogether $10.2 \%$ of jumpers had a past history of schizophrenia. Suicide by jumping is significantly more common in the Bristol and District Health Authority $(9 \cdot 3 \%$ of all suicides; $95 \%$ CI $7 \cdot 6 \%, 11 \cdot 3 \%$ ) than in England and Wales (4.9\% of suicides).

Psychiatry of Old Age Clinical Research Unit, Frenchay Healthcare NHS Trust,

Cossham Hospital, Kingswood, Bristol BS15 1LF $M$ Nowers

Department of Social Medicine, Canynge Hall, Whiteladies Road, Bristol BS8 2PR D Gunnell

Correspondence to: Dr M Nowers.

Accepted for publication July 1995
Conclusions - Patterns of suicide in the Bristol and District Health Authority are affected by the presence of the Clifton Suspension Bridge. Those who commit suicide by jumping from the bridge do not differ significantly from those using other methods of suicide. Provision of safety measures on the bridge may lead to the prevention of some suicides.

( $(\mathcal{F}$ Epidemiol Community Health 1996;50:30-32)

In $1991,5 \%(n=290)$ of all suicides in England and Wales were as a result of jumping from a height and most of these jumps were from man made structures and residential sites. ${ }^{1}$
Within the County of Avon, suicide from the Clifton Suspension Bridge in Bristol has attracted much publicity. The bridge is 245 feet above the River Avon and the chances of survival after a fall are minimal. Hence, there has been considerable discussion as to whether placing a mechanical obstruction to prevent people jumping would be effective in decreasing suicide rates. These discussions are increasingly relevant given the Government's Health of the Nation strategy which includes targets for reduction in suicide by the year 2000 .

There is evidence that making commonly used methods of suicide less accessible reduces not only the method specific but also the overall suicide rates. ${ }^{2}$ Many argue, however, that if one site were made less accessible the suicidal individual would just substitute another site or another method. In addition, it has been suggested that those who choose to commit suicide by jumping may differ from other suicides in terms of the level of intent as falling from a height is a violent and highly lethal method. A recent paper looking at falls from Brisbane River Bridges reported a high incidence of psychotic illness among those who committed suicide by jumping and in Adelaide $38 \%$ of jumpers had a past history of schizophrenia. ${ }^{34}$

This paper describes the characteristics of those who died as a result of jumping from the Clifton Suspension Bridge between April 1974 and December 1993. We have used a casecontrol study and routinely available statistics to test two hypotheses. The first hypothesis examined is that those who commit suicide by jumping from the Clifton Suspension Bridge differ both in their demographic characteristics and psychopathology from those using other methods of suicide and may therefore be less amenable to suicide prevention by removal of access to the means. Secondly, we examine whether the proximity of place of residence to the bridge itself affects choice of suicide method among Avon residents. Implications for suicide prevention are considered.

\section{Methods}

Two sources of information were used: coroners' depositions for all deaths from the suspension bridge between 1974 and 1993 and Office of Population Censuses and Surveys (OPCS) statistics (1982-91) were examined. Structured inquiry forms were used to examine the coroners's records of all deaths from the Clifton Suspension Bridge. Controls, chosen as the next suicide death occurring matched for age (within a five year age band), sex, and 
coroner's verdict (suicide, "open", or misadventure) were found from the coroners' register and these were examined in an identical way. Information was sought on the psychiatric histories of those who jumped from the bridge and their distance of residence from the bridge. Data for all deaths from the bridge were aggregated in the analysis, whatever the coroner's verdict. Accidental falls from the bridge are unlikely as the pedestrian walkway over the bridge is protected by a waist high railing. Rates of suicide clearly vary with age and sex. Matching for age and sex was undertaken because relationships between jumping from the

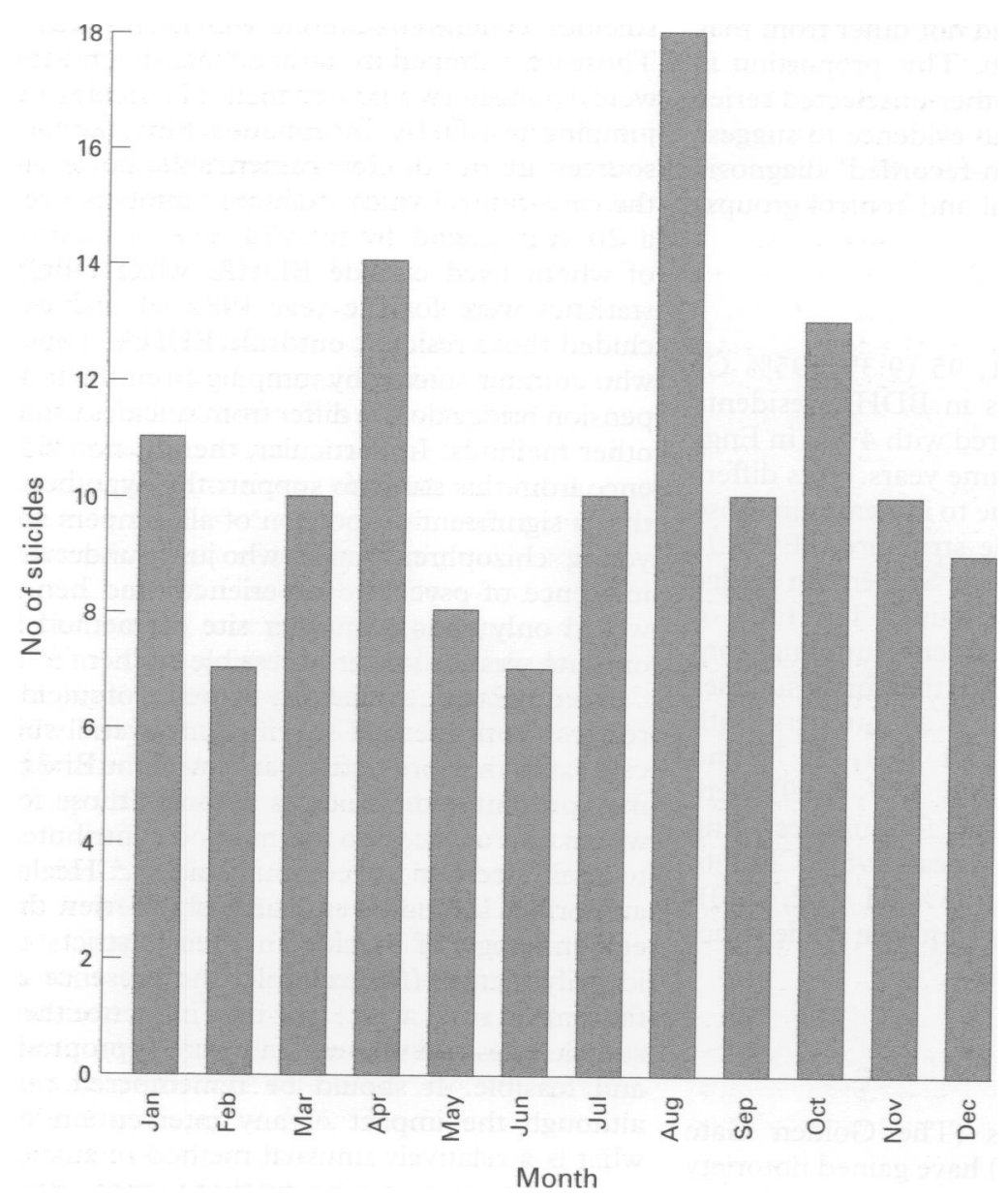

Figure 1 Clifton Suspension Bridge suicide 1974-93: month of suicide.

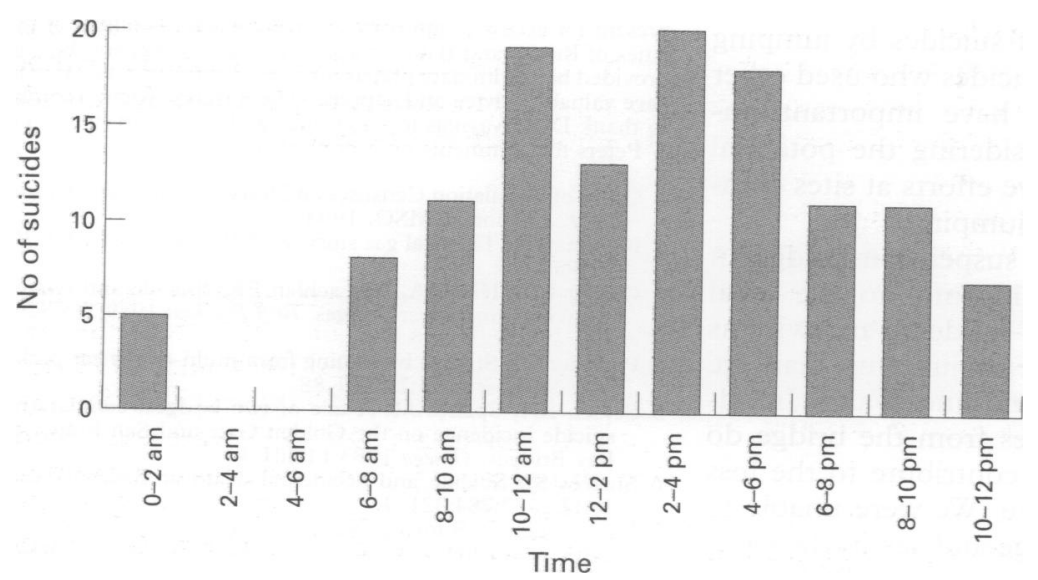

Figure 2 Clifton Suspension Bridge suicides 1974-93: time of suicide. bridge and both the factors we were interested in examining in the case-control study (past psychiatric history and proximity of residence to the bridge) may be confounded by age and sex. The potential strength of these relationships meant that we preferred to control for age and sex in the design rather than in the analysis. Although matching meant we could not examine the effects of age and sex per se in the case-control study, we were able to examine the effects of these variables using routinely available OPCS mortality data.

Data from OPCS mortality files for Bristol and District Health Authority (BDHA) 1982-91 were examined for suicide and undetermined deaths. The International Classification of Disease (ICD 9) lists suicide by jumping separately, depending on the site from which the jump occurred (residential site: E957.0/E.987.0; man made structures: E957.1/E987.1; natural sites: E957.2/E982.2; and unknown E957.9/E987.9). The geographical areas covered by the BDHA and the Avon coroner are not coterminous and therefore direct comparison of their statistics was not possible. The age and sex structure of the population of the BDHA does not differ from that of England and Wales.

McNemar's test for paired proportions; Paired, 2 sample and 1 sample $t$ tests were used for the statistical analysis.

\section{Results}

Between 1974-93 there were 127 deaths from falls from the Clifton Suspension Bridge. Coroner's verdicts of suicide $(n=96)$, "open" $(n=$ $29)$, or misadventure $(n=2)$ were returned on each of these deaths. There were no accidental deaths recorded in the period studied. There were no significant seasonal variations in suicides from the bridge nor any evidence of clustering. Most deaths (18) occurred in August (fig 1). Most jumps (81 (63.8\%)) occurred in the eight hours between 10 am and $6 \mathrm{pm}$ (fig 2). There were 93 males (mean age 35.4 years) and 34 females (mean age 35.5 years). Thirty six of the people who died (28.3\%) lived outside Avon - some as far away as Oxford, Shrewsbury and London.

\section{CASE-CONTROL STUDY}

Information on the distance of residence from the bridge and past psychiatric history was sought from the coroner's depositions for the 127 falls from the bridge and the 127 matched control deaths. For the Avon residents there was no significant difference in terms of distance to the bridge from their home address between those who used the bridge $(7.3 \mathrm{~km})$ or their matched controls who used other methods $(9.0 \mathrm{~km})(95 \%$ confidence interval (CI) for difference $-0.5,3.9 \mathrm{~km}$ ).

The psychiatric histories of all jumpers were compared with the control population (table). Although a greater proportion of jumpers $(55 \cdot 1 \%)$ had a past psychiatric history compared with the matched controls $(44 \cdot 1 \%)$, the difference did not reach conventional levels of 
Psychiatric histories and diagnoses of those who jumped from the Clifton Suspension Bridge and matched controls

\begin{tabular}{lll}
\hline & Suspension bridge jumpers $(n=127)$ & Age and sex matched controls $(n=127)$ \\
\hline Mean (SD) age (y) & Male: $35 \cdot 4$ years $(14 \cdot 9)$ & Male: $36 \cdot 2(14 \cdot 6)$ \\
& Female: $35 \cdot 5$ years $(12 \cdot 6)$ & Female: $34 \cdot 8(12 \cdot 3)$ \\
No with a psychiatric history (\%) & $70(55 \cdot 1)$ & $56(44 \cdot 1)$ \\
No who have been psychiatric inpatients (\%) & $46(36 \cdot 2)$ & $44(34 \cdot 6)$ \\
Main recorded psychiatric diagnoses (\%) & Depression: $48(37 \cdot 8)$ & Depression: $33(26)$ \\
& Schizophrenia: $13(10 \cdot 2)$ & Schizophrenia: $11(8 \cdot 7)$ \\
& None recorded: $52(40 \cdot 9)$ & None recorded: $71(55 \cdot 9)$ \\
\hline
\end{tabular}

statistical significance $(95 \% \mathrm{CI}$ of the difference $-1 \cdot 17,23 \cdot 2 \%)$. Similar proportions of jumpers and control suicides had been psychiatric inpatients in the past; $46(36.2 \%)$ of jumpers and $44(34.6 \%)$ of controls had such a history. The proportion of jumpers with a history of schizophrenia $(10 \cdot 2 \%)$ did not differ from that in the control population. This proportion is similar to that found in other unselected series of suicides. ${ }^{7}$ There was no evidence to suggest any difference in the "non-recorded" diagnosis between the experimental and control groups.

\section{OPCS MORTALITY DATA}

Between 1982 and 1991, 95 (9.3\%; 95\% CI $7 \cdot 6,11 \cdot 3)$ of all suicides in BDHA residents were by jumping, compared with $4.9 \%$ in England and Wales for the same years. This difference is almost entirely due to increases in those jumping from man made structures (E957.1; E987.1) of which the Clifton Suspension Bridge is by far the major contributor. The ratio of male to female BDHA residents jumping from a height was $1 \cdot 7: 1$, similar to that for all suicides $(2 \cdot 1: 1)$ The mean ages of all jumpers both male: $42 \cdot 2$ years $(95 \%$ CI $33.1,51 \cdot 7)$ and female 44.8 years $(95 \%$ CI $32.7,56.9)$ were not significantly lower than for suicides using other methods - male $44 \cdot 0$ years $(95 \%$ CI $42 \cdot 6$, $45 \cdot 4)$, female $51 \cdot 6$ years $(95 \%$ CI $49 \cdot 2,54 \cdot 0)$. However, males jumping from man made structures tended to be younger.

\section{Discussion}

A number of locations (The Golden Gate Bridge, the Eiffel Tower) have gained notoriety as popular places from which to jump, ${ }^{35}$ and in England and Wales beauty spots such as Beachy Head have been the site of many suicides in the past. ${ }^{6}$ However, this is the first description of a series of suicides by jumping compared to matched suicides who used other methods. The findings have important implications for those considering the potential effectiveness of preventive efforts at sites commonly used for suicidal jumping.

The presence of the suspension bridge in Bristol contributes significantly to the local pattern of suicide. Local residents are twice as likely to commit suicide by jumping than are other residents in England and Wales. In addition, a third of suicides from the bridge do not live locally but still contribute to the loss of life from this structure. We were unable to examine the effects of age and sex in the casecontrol study. However, routine OPCS mor- tality statistics for BDHA showed that residents who committed suicide by jumping, whether from the suspension bridge or elsewhere, were similar in age and sex distribution to those who used other methods of suicide. Jumps from the suspension bridge account for half of the suicides by jumping among BDHA residents. Those who jumped from the suspension bridge were younger as a group than all suicides by jumping in BDHA. Information from the two sources are not directly comparable, however; the case-control study examined jumpers over a 20 year period from 1974, over a quarter of whom lived outside BDHA, while OPCS statistics were for the year 1982-91 and excluded those resident outdside BDHA. Those who commit suicide by jumping from the suspension bridge do not differ from suicides using other methods. In particular, there is no evidence from this study to support the hypothesis that a significant proportion of all jumpers are young schizophrenic males who jump under the influence of psychotic experiences and hence would only choose another site or method if one site was no longer accessible to them. ${ }^{36}$

If reducing the availability of means of suicide reduces both method specific and overall suicide rates, then protective barriers on the Bridge may prevent some suicides amongst those for whom ease of access to this method contributed to their decision to commit suicide. ${ }^{2}$ Health authorities should be encouraged to review the epidemiology of suicide in their districts to identify factors (for example, the presence of firearms in rural areas) that may influence their suicide rates and take action where appropriate and feasible. It should be remembered that although the impact of any intervention on what is a relatively unusual method of suicide such as jumping may be difficult to measure in statistical terms, it may be of immeasurable benefit in human terms.

The authors wish to thank HM Coroners for Avon past and present for access to information and the records offices of the cities of Bristol and Bath for access to records. Simeon Barron provided help with data preparation and Professor HG Morgan gave valuable advice and support. The authors would also like to thank Dr C Vassilas for assistance and Dr I Harvey and D T Peters for comments on a draft of this paper.

1 Office of Population Censuses and Surveys. Mortality statistics 1991. London: HMSO, 1993.

2 Kreitman N. The coal gas story. Br $\mathcal{F}$ Prev Soc Med 1976;30: 86-93.

3 Cantor $\mathrm{CH}$, Hill MA, McLachlan EK. Suicide and related behaviour from river bridges. $B r \mathcal{F}$ Psychiat 1989;155:829 35.

4 Pounder DJ. Suicide by leaping from multi-storey car parks Med Sci Law 1985;25:179-88.

5 Seiden $\mathrm{RH}$, Spence $M$. A tale of two bridges: comparative suicide incidence on the Golden Gate and San Francisco Bay Bridges. Omega 1983;14:201-9.

6 Surtees SI. Suicide and accidental death at Beachy Head. BMF 1982;284:321-4.

7 Barraclough B, Bunch J, Nelson B, Sainsbury P. A hundred cases of suicide: clinical aspects. Br $\mathcal{F}$ Psychiat 1974;125: 355-73. 